\title{
Chaotic ratchet dynamics with cold atoms in a pair of pulsed optical lattices
}

\author{
Gabriel G. Carlo, ${ }^{1,2}$ Giuliano Benenti, ${ }^{1,3}$ Giulio Casati, ${ }^{1,3,4}$ Sandro Wimberger, ${ }^{5}$ Oliver Morsch, ${ }^{5}$ \\ Riccardo Mannella, 5 and Ennio Arimondo 5 \\ ${ }^{1}$ Center for Nonlinear and Complex Systems, Università degli Studi dell'Insubria, Via Valleggio 11, 22100 Como, Italy \\ ${ }^{2}$ Departamento de Física, Comisión Nacional de Energía Atómica, Avenida del Libertador 8250, 1429 Buenos Aires, Argentina \\ ${ }^{3}$ Istituto Nazionale di Fisica Nucleare, Sezione di Milano and CNISM, and CNR-INFM, Via Celoria 16, 20133 Milano, Italy \\ ${ }^{4}$ Department of Physics, National University of Singapore, Singapore 117542, Republic of Singapore \\ ${ }^{5}$ CNR-INFM and Dipartimento di Fisica "Enrico Fermi," Università degli Studi di Pisa, Largo Pontecorvo 3, 56127 Pisa, Italy
}

(Received 29 May 2006; published 22 September 2006)

\begin{abstract}
We present a very simple model for realizing directed transport with cold atoms in a pair of periodically flashed optical lattices. The origin of this ratchet effect is explained and its robustness demonstrated under imperfections typical of cold atom experiments. We conclude that our model offers a clear-cut way to implement directed transport in an atom optical experiment.
\end{abstract}

DOI: $10.1103 /$ PhysRevA.74.033617

PACS number(s): 03.75.Be, 05.45.Mt, 05.40.Jc, 32.80.Pj

\section{INTRODUCTION}

The atom optics realization of the paradigmatic kicked rotor (KR) [1] presents the possibility to study experimentally unique quantum mechanical aspects of a fundamental, classically nonlinear system. Dynamical Localization is perhaps the most celebrated quantum phenomenon observed in the quantum KR [1,2], but many other interesting features of the KR have been studied theoretically and experimentally $[3,4]$. Very recently, applications of modified KR models have been designed which allow for a controlled, directed motion of particles in momentum space $[5,6]$.

The atom-optics kicked rotor (AOKR) is realized by subjecting cold atoms $[3,4]$ or a Bose condensate $[7-10]$ to a far detuned standing wave with spatial period $\pi / k_{L}$ ( $k_{L}$ being the wave number of the kicking laser) and pulsed with period $\tau$. The AOKR is described, in dimensionless units, by the Hamiltonian [11]

$$
\mathcal{H}(t)=\frac{p^{2}}{2}+k \cos (x) \sum_{n=0}^{\infty} \delta(t-n T)
$$

where $p$ is the atomic momentum in units of $2 \hbar k_{L}$ (i.e., of two-photon recoil momenta), $x$ is the atomic position divided by $2 k_{L}, t$ is time, and $n$ is an integer which counts the kicks. Experimentally, $\delta$ kicks are approximated by pulses of width $\tau_{p}$ which are approximately rectangular in shape. We also define an effective Planck's constant $\hbar_{\text {eff }}=T=8 E_{R} \tau / \hbar$, where $E_{R}=\left(\hbar k_{L}\right)^{2} / 2 M$ is the recoil energy (acquired by an atom after emission of a photon with wave number $k_{L}$ ). The dimensionless parameter $k \approx V_{0} \tau_{p} / \hbar$ is the kicking strength of the system (with $V_{0}$ the height of the optical lattice creating the kicking potential).

In this paper, we propose a ratchet which could be realized experimentally by adding to the standard AOKR dynamics defined by Eq. (1) a second kicking potential (applied in a synchronized way with respect to the first one). The application of a second kicking potential to the atom has some analogy with the double AOKR investigated in Ref. [12], because in both cases a sequence of two kicks is applied to the atoms. In the present investigation a spatial shift of the second kick potential is also included. We show that this is sufficient to produce the ratchet effect. Moreover, we consider the effects of a particle escape mechanism similar to evaporative cooling [13]. More precisely, we study an open system with absorbing boundary conditions. If $\psi(p)$ is the wave function in momentum space, absorbing boundary conditions are implemented by the prescription $\psi(p) \equiv 0$ if $p \leqslant$ $-p_{c}$ or $p \geqslant p_{c}$. Such absorbing boundary conditions could be realized experimentally using, e.g., velocity selective Raman transitions, which change the internal states of the atoms, and hence let them escape from the states of interest [14], or by other state selective methods [15]. Such a scenario of loosing the faster atoms with momenta exceeding $p_{c}$, is analogous to evaporative cooling of cold atoms [13]. The time scale of the applied absorption mechanism should be of the order of the kicking period $T$ to allow for a steady loss of atoms during the system's evolution.

We point out that, as shown below, in our model the ratchet phenomenon is also present in the Hamiltonian limit without escape of particles. On the other hand, it is interesting to investigate the particle escape mechanism because it models the evaporative cooling process natural in cold atoms experiments. Moreover, its introduction is relevant in order to analyze the stability of our proposed ratchet mechanism after that atoms excited to higher and higher velocities by chaotic diffusion are eventually lost. Finally, particle escape may allow the unprecedented experimental observation of a quantum phase space distribution located on an underlying classical fractal set.

In state-of-the-art atom optics experiments, control over the kicking strength $k$ (or, equivalently over the laser power delivered to the atoms) is achieved with a precision of a few percent [4]. Kicking strengths in the range 1-7 correspond to standing wave amplitudes of about 80-600 $E_{R}$ for rubidium atoms (and assuming a rectangular pulse shape with a width of $500 \mathrm{~ns}$ ). Below we will be interested in the parameter region of small kicking periods $T \leqq 1$, and hence it is important to note that time is one of the best controlled experimental parameters, and kicking periods between about one hundred nanoseconds and a few hundred microseconds are available, with a maximal precision of a few tens of nano- 
seconds $[4,16,17]$. For cesium atoms, this range corresponds to dimensionless kicking periods $T \approx 10^{-2}-20$, and a maximal precision of $\delta T \geqslant 10^{-3}$ [17]. Atom optics experiments may be performed on two different atomic samples: laser cooled atoms and Bose-Einstein condensates. The main difference is the initial width $\Delta p_{0}$ in momentum. For laser cooled atoms and in the best conditions, the initial width in momentum corresponds to a few two-photon recoils units. For Bose-Einstein condensates $\Delta p_{0}$ between 0.01 and 0.05 can be realized $[7,8,10,18]$. Bose-Einstein condensates experience a nonlinear potential associated with the atom-atom interaction. However, letting the condensate expand a little before the actual kicking evolution allows one to reduce the atom-atom interactions to negligible values, with only slight changes in $\Delta p_{0}$ [7]. The present analysis focuses on a sample of laser cooled atoms with a large initial momentum distribution. In fact, this condition is more favorable for the realization of the ratchet discussed in this paper, because the sample explores a larger region of the classical phase space and therefore exploits the structure of phase space (a strange repeller, in the classical limit) induced by the evaporative cooling process.

The paper is organized as follows. Section II analyzes the AOKR model and its evolution in phase space under the double kicking perturbation. Section III investigates different imperfections associated with the experimental realization. For instance, a deep optical potential is required for lasercooled atoms, and in such conditions spontaneous emissions become a non-negligible issue. In addition, fluctuations in the laser power and other sources of noise are included in the analysis. The final Sec. IV concludes with an outlook discussing the role of nonlinearity as present in experiments using a Bose-Einstein condensate.

\section{MODEL AND PROPERTIES}

In this section we introduce a kicked system that shows directed transport and in which the direction of the current can be controlled. This is done in a very simple way, we just have to duplicate the series of kicks in Eq. (1) in a convenient fashion. This simplicity is essential for an efficient experimental implementation with cold atoms.

We consider a particle moving in one dimension $[x \in(-\infty,+\infty)]$ in a periodically kicked potential. The Hamiltonian reads

$$
\begin{gathered}
\mathcal{H}_{2}(t)=\frac{p^{2}}{2}+V_{\phi, \xi}(x, t), \\
V_{\phi, \xi}=k \sum_{n=-\infty}^{+\infty}[\delta(t-n T) \cos (x)+\delta(t-n T-\xi) \cos (x-\phi)],
\end{gathered}
$$

where $T$ is the kicking period. In fact, we propose an asymmetric kicking sequence. This is made out of two series of kicks with the same spatial and temporal periods, $2 \pi$ and $T=2 \pi / \omega$, but shifted by a phase $\phi(0 \leqslant \phi<2 \pi)$ and a time $\xi$
$(0 \leqslant \xi<T)$. Due to the spatial periodicity of the kicking potential $V_{\phi, \xi}$, the one-cycle evolution (Floquet) operator

$$
\hat{\mathcal{U}}=e^{-i(T-\xi) \hat{p}^{2} / 2} e^{-i k \cos (\hat{x}-\phi)} e^{-i \xi \hat{p}^{2} / 2} e^{-i k \cos (\hat{x})}
$$

induced by the Hamiltonian of Eq. (2) commutes with spatial translations by multiples of $2 \pi$. As is well known from Bloch theory, this implies conservation of the quasimomentum $\beta$, defined as the fractional part of the momentum $p(0$ $\leqslant \beta<1$ ) [19]. For a given value of the quasimomentum, the wave function of the system is a Bloch wave, of the form $e^{i \beta x} \psi_{\beta}(x)$, where $\psi_{\beta}(x)$ is a function of period $2 \pi$. A generic wave function can then be written as a superposition of Bloch waves $\psi(x)=\int_{0}^{1} d \beta e^{i \beta x} \psi_{\beta}(x)$.

Introducing the rescaled momentum variable $I=T p$, one can see that classical dynamics of model (2) depends on the scaling parameter $K=k T$ (not on $k$ and $T$ separately). The classical limit corresponds to $\hbar_{\text {eff }}=T \rightarrow 0$, while keeping $K$ $=\hbar_{\text {eff }} k$ constant.

In order to simulate the evaporative cooling process in the quantum model we consider the projection over a subspace corresponding to the quantum levels that are below $p_{c}$ (in absolute value). In practice, this is implemented at each kick: if we denote by $\hat{P}$ the projection operator on the interval ]$-p_{c}, p_{c}[$, the wave function after $n$ kicks is then given by

$$
\psi(p, n)=(\hat{P} \hat{\mathcal{U}})^{n} \psi(p, 0) .
$$

Note that quasimomentum is still a conserved quantity. In the classical case, we consider lost the particles that reach momentum $p$ such that $|p|>p_{c}$.

We have checked in our numerical simulations that the dependence of the ratchet current on the cutoff value $p_{c}$ is weak, provided that $p_{c} \gg k$. Therefore, the ratchet current in this regime turns out to be close to the current obtained in the Hamiltonian limit $p_{c} \rightarrow \infty$. On the other hand, the particle escape mechanism strongly affects the phase space structure, leading, in the classical limit, to the setting in of a strange repeller.

In the numerical simulations reported in this paper, we fix $K=7$, corresponding to the classically chaotic regime, $\xi$ $=T / 3$, and $p_{c} \hbar_{\mathrm{eff}}=15.2$. The initial state is given by a uniform mixture of the momentum states inside the interval $p \hbar_{\mathrm{eff}} \in[-1,1]$. Once the quasi-momentum is fixed, the number of momentum states in this interval is $\propto 1 / \hbar_{\text {eff }}$. Moreover, we average numerical data over $10^{3}$ randomly chosen quasimomenta. Classical averages are constructed from $10^{7}$ initial conditions randomly and uniformly distributed inside the region $x \in[0,2 \pi), I=p T \in[-1,1]$. Note that with these initial conditions and the above parameter values we are left with approximately $35 \%$ of the initial number of particles at time $n=t / T=10$ and $10 \%$ at $n=20$.

The appearance of a strange repeller in our model in the classical limit is shown in the phase space portrait of Fig. 1 (a), obtained for $\phi=\pi / 2$ at $n=20$. The three panels of Fig. 1 correspond, from (a) to (c), to the classical Poincare section and the quantum Husimi function at $\hbar_{\mathrm{eff}} \simeq 0.16$ and $\hbar_{\mathrm{eff}} \simeq 1$. We can see a good agreement between the classical and the quantum phase space portraits. Quantum fluctuations smooth 


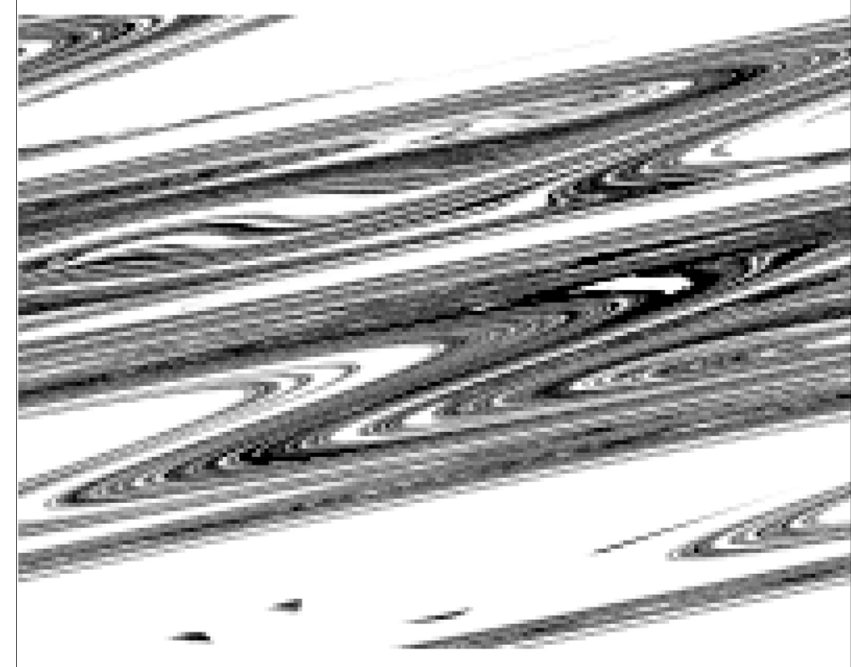

(a)

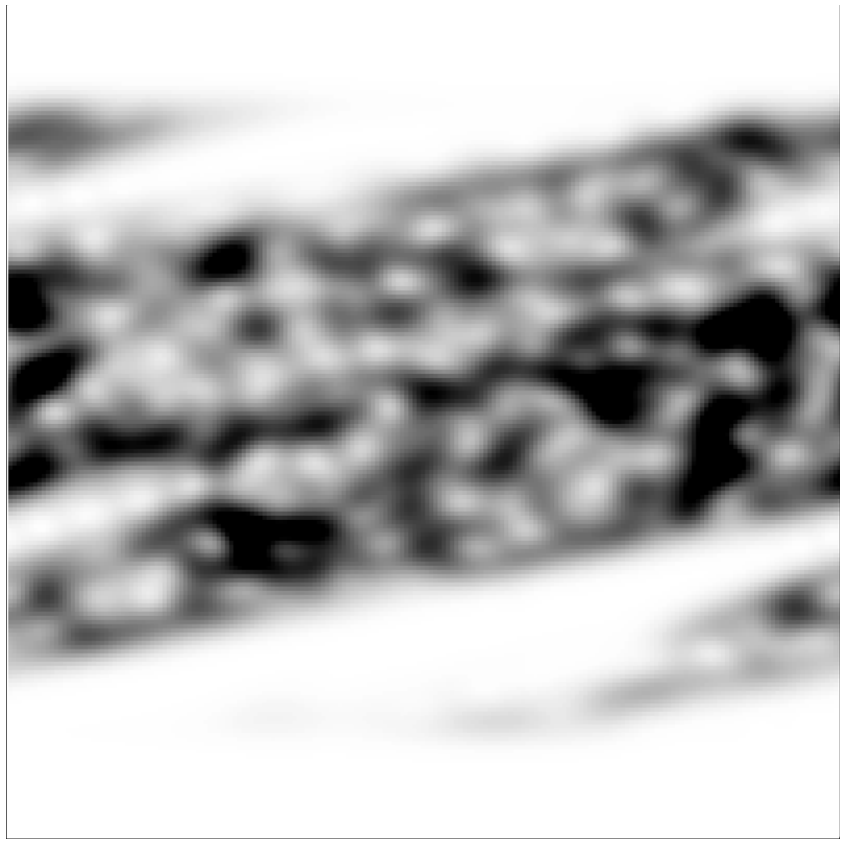

(b)

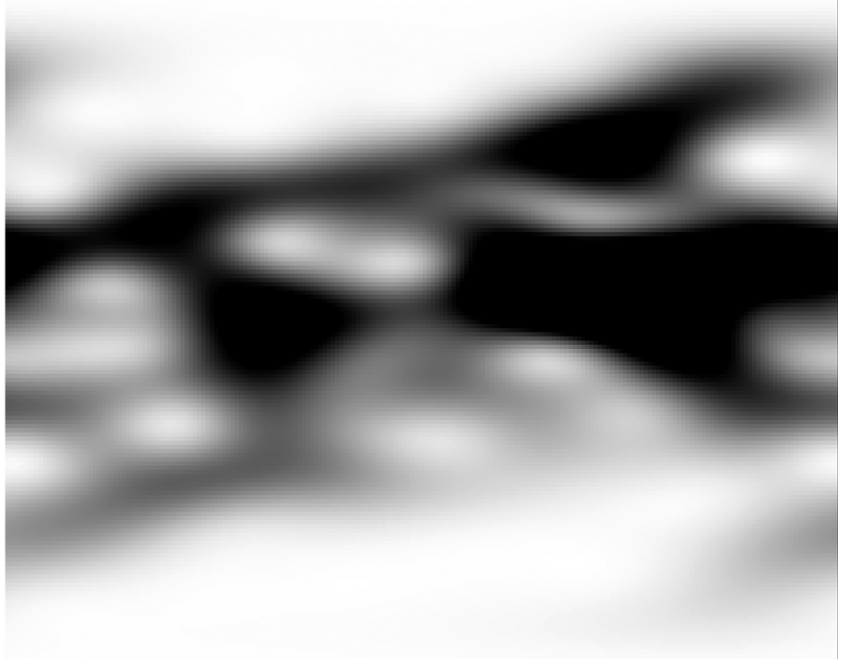

(c)

FIG. 1. Phase space pictures for $\phi=\pi / 2$, at $n=20$ : classical Poincaré sections (a) and quantum Husimi functions at $\hbar_{\mathrm{eff}} \simeq 0.16$ (b) and $\hbar_{\mathrm{eff}} \simeq 1$ (c). The displayed region is given by $I=p T \in[-20,20]$ (vertical axis) and $x \in[0,2 \pi)$ (horizontal axis). Note that, to draw the attractor, $x$ is taken modulus $2 \pi$. The brightness is inversely proportional to the density: black (white) regions correspond to maximal (zero) density.

the fractal structure of the classical repeller on the scale of Planck's cell [20]. In the quantum case the values of $\hbar_{\text {eff }}$ $\simeq 0.16$ and $\hbar_{\text {eff }} \simeq 1$ considered here (and suitable for a realistic experimental implementation) are not sufficiently small to resolve the fractal structure at small scales. However, the Husimi function shows clear similarities with the underlying classical probability distribution. Even for $\hbar_{\mathrm{eff}}=T \simeq 1$ the major features of the classical repeller (i.e., width in phase space and asymmetry) are visible. Parameter values and evo- lution time are suitable for the experimental measurement of the quantum probability distribution located on the underlying classical strange repeller. This is important because the appearance of strange sets (attractors or repellers) is a distinctive feature of open chaotic systems.

The repeller in Fig. 1 is strongly asymmetric, suggesting directed transport, that is, $\langle p\rangle \neq 0$. This is confirmed by the numerical data of Fig. 2, where $\langle p\rangle$ is shown as a function of the time $n$. 


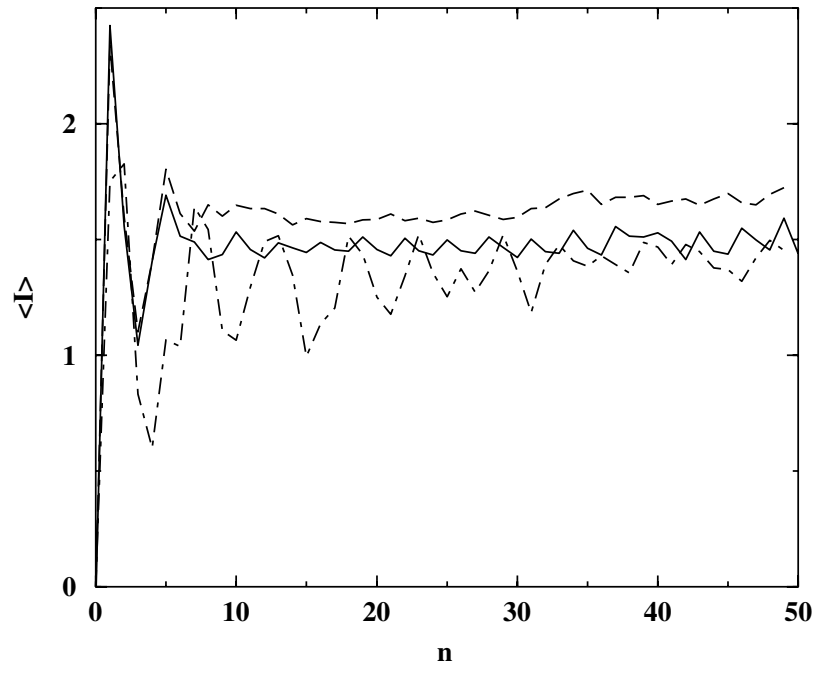

FIG. 2. Average rescaled momentum $\langle I\rangle=\langle p\rangle T$ as a function of the discrete time $n$, for the same parameter values as in Fig. 1 . The solid curve corresponds to the classical case, while the dashed curve corresponds to quantum results for $\hbar_{\mathrm{eff}} \simeq 0.16$ and the dot-dashed one to $\hbar_{\text {eff }} \simeq 1$.

We can explain the origin of the directed current present in our system by following the approach developed in Ref. [21]. We have a classical time evolution given by

$$
\ddot{x}+f_{\phi, \xi}(x, t)=0,
$$

where $f_{\phi, \xi}(x, t)=\partial V_{\phi, \xi}(x, t) / \partial x$. To this equation we add a particle escape process consisting of cutting out the orbits that exceed a given value of the momentum $p=\dot{x}$. We are interested in symmetry transformations that leave Eq. (5) invariant but change the sign of $p$. In fact, if we assume that our system is chaotic we can generate for each orbit its $p$-reversed partner, which will explore the whole region embedding the chaotic trajectories. This amounts to saying that, being essentially equivalent, both orbits (and all of them) should have zero average momentum. If these symmetries are absent it is natural to conclude that a net $p$ (i.e., different from zero) can be generated. Thus, breaking all possible symmetries of this kind constitutes a good method to engineer ratchet systems. As the particle escape process introduced above is symmetrical with respect to $p$, we can neglect it in the following reasoning. It is worth mentioning that all the symmetry considerations developed in this section translate almost immediately to the quantum case.

There are two general ways to change the sign of $p$ :

$$
\text { (I) } x \rightarrow-x+\alpha, \quad t \rightarrow t+\gamma
$$

and

$$
\text { (II) } x \rightarrow x+\alpha, \quad t \rightarrow-t+\gamma \text {. }
$$

In order to leave Eq. (5) unchanged we need that $f_{\phi, \xi}(x, t)$ $=-f_{\phi, \xi}(-x+\alpha, t+\gamma)$ holds for (I), since $\ddot{x} \rightarrow-\ddot{x}$ under this transformation. If we apply twice transformation (I) we obtain $f_{\phi, \xi}(x+\alpha / 2, t)=f_{\phi, \xi}(x+\alpha / 2, t+2 \gamma)$. Since $f_{\phi, \xi}(x, t)$ is assumed to be bounded and periodic with zero mean, both in $x$ and $t, \gamma$ can only be an integer multiple of $T / 2$ (including the $\gamma=0$ case). In turn, there are no restrictions on $\alpha$. On the other hand, for (II) we need $f_{\phi, \xi}(x, t)=+f_{\phi, \xi}(x+\alpha,-t+\gamma)$ (with a plus sign since now $\ddot{x}$ keeps its original sign). By applying twice transformation (II) we obtain $f_{\phi, \xi}(x, t+\gamma / 2)$ $=f_{\phi, \xi}(x+2 \alpha, t+\gamma / 2)$. Following the same reasoning as before, $\alpha$ is fixed to integer multiples of $\pi$ (including $\alpha=0$ ) while there are no restrictions on $\gamma$. Note that (I) and (II) are the only two symmetries that should be broken in order to find directed transport. Our choice of the potential (2) guarantees the possibility to break both of them.

In fact, we have that $f_{\phi, \xi}(x, t)=k \sum_{-\infty}^{+\infty}[-\delta(t-n T) \sin (x)$ $-\delta(t-n T-\xi) \sin (x-\phi)]$, and in the case of symmetry (I) we require that $f_{\phi, \xi}(x, t)=-f_{\phi, \xi}(-x+\alpha, t+\gamma)$. We can take $\gamma=0$ without loss of generality since we only have a sum of delta functions in $t$, i.e., the sign change of $f$ induced by symmetry (I) can only come from the first part of the transformation $(x \rightarrow-x+\alpha)$. Therefore, we arrive at the conditions $\sin (-x$ $+\alpha)=-\sin (x)$ and $\sin (-x+\alpha-\phi)=-\sin (x-\phi)$. These two conditions lead to $\alpha=l 2 \pi$ and $\alpha=l^{\prime} 2 \pi+2 \phi$, with $l$ and $l^{\prime}$ integers, and cannot be fulfilled together, except for $\phi=0$ or $\phi=\pi$. Therefore, symmetry (I) is broken when $\phi \neq 0, \pi$.

In the case of symmetry (II), if we take $\alpha$ an odd multiple of $\pi$ then the sign of $f_{\phi, \xi}$ changes. Then, we are only left with $\alpha$ being an even multiple of $\pi$, i.e., we can take $\alpha=0$ without loss of generality. Moreover, we notice that if $\phi=0$ and $\alpha=0$ both kicks become the same in $x$ and therefore symmetry (II) holds for any $\xi$, taking $\gamma=\xi$. On the other hand, considering $\phi \neq 0$ we arrive at the conditions $\Sigma_{n=-\infty}^{+\infty} \delta(-t+\gamma-n T)=\Sigma_{n=-\infty}^{+\infty} \delta(t-n T)$ and $\sum_{n=-\infty}^{+\infty} \delta(-t+\gamma-n T$ $-\xi)=\sum_{n=-\infty}^{+\infty} \delta(t-\xi)$, which imply $\gamma=l T$ and $\gamma=l^{\prime} T+2 \xi$, with $l$ and $l^{\prime}$ integers. We conclude that, if $\phi \neq 0$, symmetry (II) is broken when $\xi \neq 0, T / 2$.

In summary, both symmetries (I) and (II) are broken for $\phi \neq 0, \pi$ and $\xi \neq 0, T / 2$. Hence two series of kicks are sufficient to observe the ratchet effect, provided that these kicks are shifted both in space and in time, the shift in space being different from half wavelength and the shift in time being different from half period.

It is interesting to remark that current reversal can be engineered in a very simple way, by taking $\widetilde{\phi}=-\phi$ instead of $\phi$ in Eq. (2). Indeed, Eq. (5) is left unchanged when $x \rightarrow$ $-x, t \rightarrow t$, and $\phi \rightarrow \widetilde{\phi}=-\phi$, while this transformation changes the sign of $p$. We can see current inversion in Fig. 3, both in the classical and in the quantum case, when $\phi=\pi / 2 \rightarrow \widetilde{\phi}=$ $-\pi / 2$. Note that $\langle p\rangle=0$ at $\phi=0$, in agreement with the above symmetry considerations.

\section{STABILITY OF THE RATCHET EFFECT UNDER IMPERFECTIONS}

The purpose of this section is to study the robustness of the ratchet effect introduced in this paper in the presence of typical sources of noise in cold-atom experiments. For the large kicking strengths needed to guarantee clear signatures of a chaotic repeller, spontaneous emission during the flashing of the optical lattice cannot be ruled out [3]. Spontaneous emission can be effectively modeled by random jumps in quasimomentum [19]. We test the influence of such random 


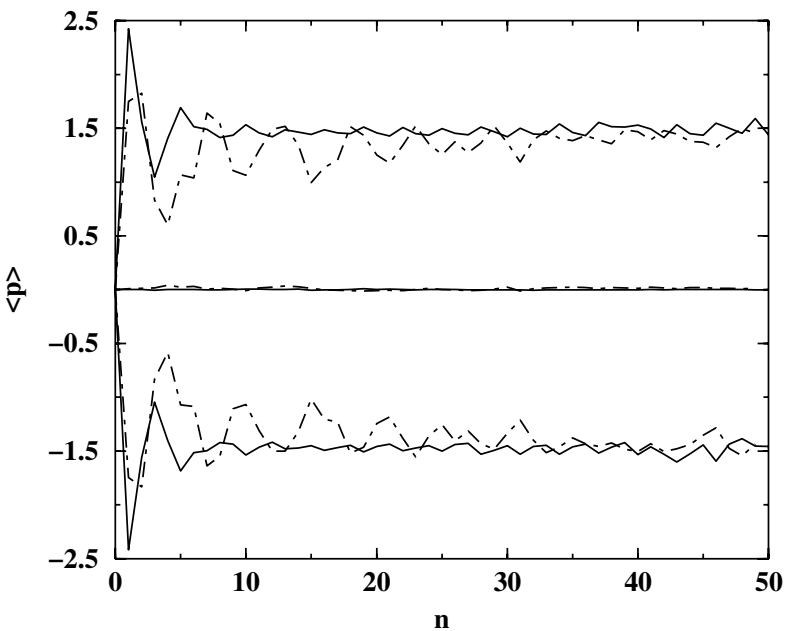

FIG. 3. Average momentum $\langle p\rangle$ as a function of $n$, for $\phi$ $=\pi / 2$ (positive values), $\phi=0$ (zero values), and $\phi=-\pi / 2$ (negative values). Both the classical (solid curves) and the quantum case (dotdashed curves, $\hbar_{\text {eff }} \simeq 1$ ) are shown. Note that at $\phi=0$ quantum and classical curves are almost superimposed.

changes in quasimomentum on the results presented in the previous section. That is to say, we repeat the previous calculations but letting at any kick the quasimomentum randomly change with a probability of $0,0.2$, and 0.5 (see Fig. 4). In practice, it may jump to any possible value in the Brillouin zone with those probabilities. As can be seen, this additional randomness even helps to reduce fluctuations, and when the jump probability is different from zero there is a better convergence towards the classical result.

We now investigate how different kind of errors affect the value of the ratchet current. More precisely, we compute the average current $\langle p\rangle_{\mathrm{av}}$, obtained after averaging $\langle p\rangle$ in the time interval $10 \leqslant n \leqslant 20$, as a function of the noise strengths associated to different noise sources.

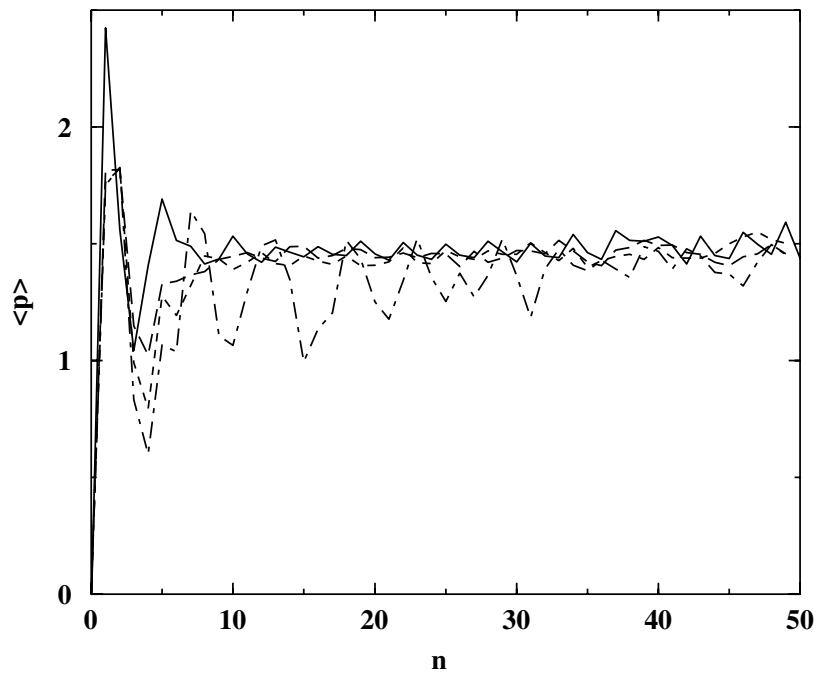

FIG. 4. Average momentum $\langle p\rangle$ as a function of the discrete time $n$, for the same parameters as in Fig. 2 , at $\hbar_{\text {eff }} \simeq 1$. At each kick, the quasimomentum can jump to any other possible value with probabilities 0 (dot-dashed curve), 0.2 (dashed curve), and 0.5 (long dashed curve). The solid curve corresponds to the classical case.

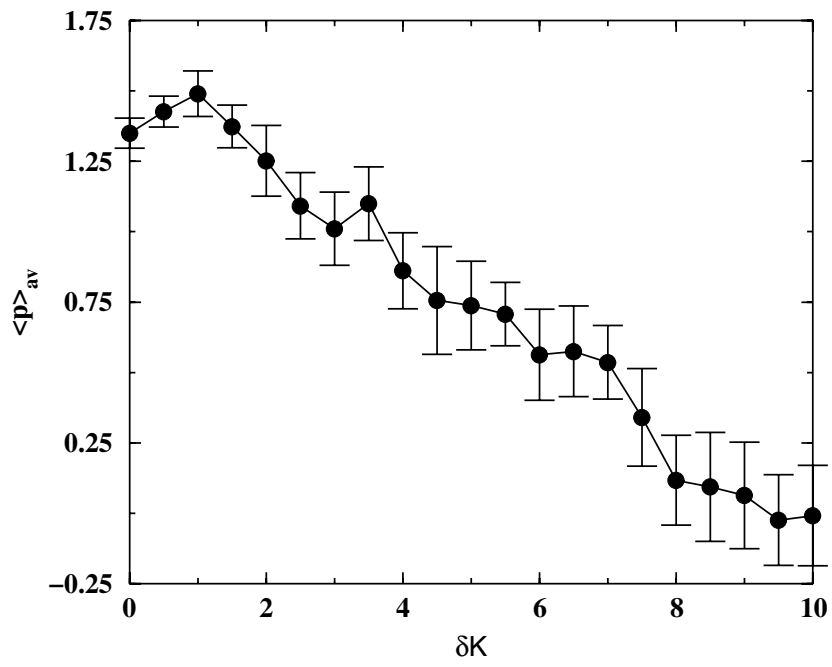

FIG. 5. Average current $\langle p\rangle_{\mathrm{av}}$ as a function of the noise $\delta K$ in the kick strength $K$, for parameter values as in Fig. 2 , at $\hbar_{\mathrm{eff}} \simeq 1$.

First of all, we consider the effects of fluctuations in the kicking strength. This is simulated by memoryless random errors of size $\delta K$ in the value of $K$ : the kicking strength $K_{n}$ at time $n$ is given by $K_{n}=K+(\delta K)_{n}$ where the noise value $(\delta K)_{n}$ is randomly drawn from a uniform distribution in the interval $[-\delta K, \delta K]$. It can be seen in Fig. 5 that the ratchet effect is stable up to approximately $\delta K \simeq 2$, corresponding to a relative amplitude noise of $\delta K / K \simeq 0.3$.

Since the ratchet mechanism described in the previous section works the better the smaller we choose $\hbar_{\mathrm{eff}}=T$, we consider possible fluctuations in the kicking period [16] arising from the problem of controlling strong but narrow pulses in time with a high repetition rate. We model these imperfections as random and memoryless fluctuations in the period between consecutive kicks. This takes into account the fact that the timing of the kicks can suffer from uncontrollable variations. As we can see from Fig. 6, stability is quite sat-

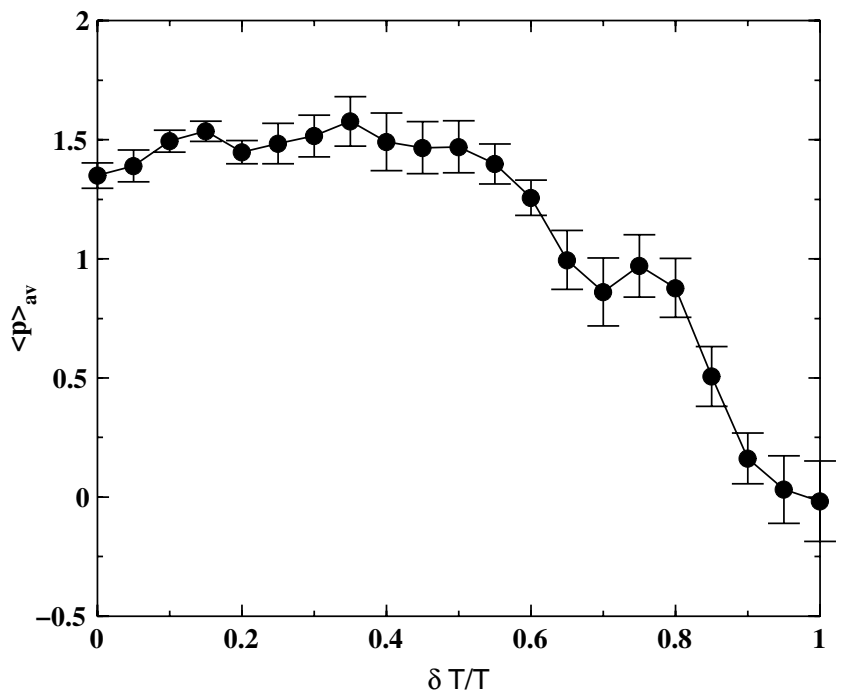

FIG. 6. Average current $\langle p\rangle_{\mathrm{av}}$ as a function of the relative error $\delta T / T$ in the kicking period $T$, for parameter values as in Fig. 5. 


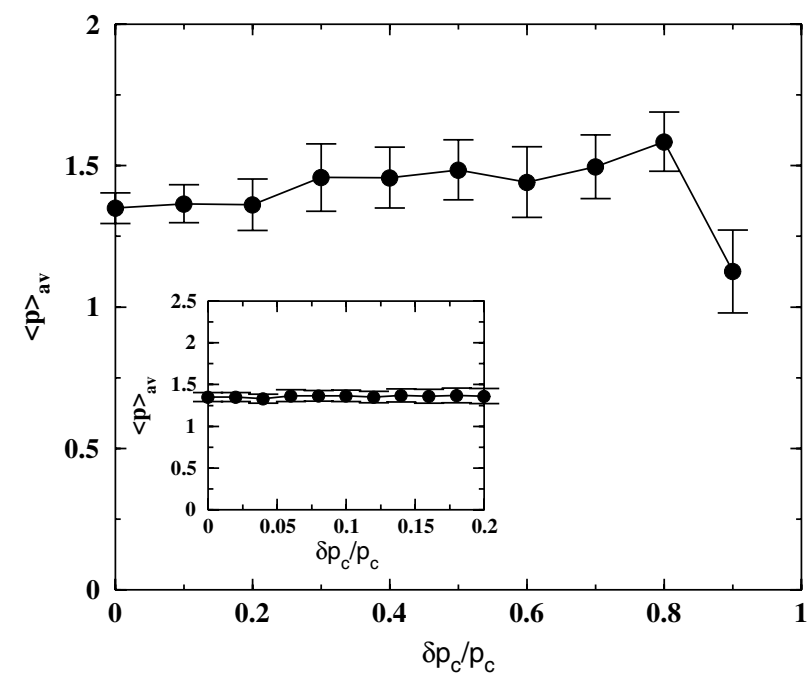

FIG. 7. Average current $\langle p\rangle_{\mathrm{av}}$ as a function of the relative error $\delta p_{c} / p_{c}$ in the cutoff momentum $p_{c}$, for parameter values as in Fig. 5. A magnification of the figure for small values of $\delta p_{c} / p_{c}$ is shown in the inset.

isfactory when $\delta T / T \leqq 0.5$, where $\delta T$ is the size of the fluctuations and $T \simeq 1$.

Finally, we consider the effect of an imprecision in the $p_{c}$ selection. This is modeled by random memoryless variations of the cutoff value $\left(p_{c}\right)_{n}$ used at time $n:\left(p_{c}\right)_{n}=p_{c}+\left(\delta p_{c}\right)_{n}$, with $\left(\delta p_{c}\right)_{n} \in\left[-\delta p_{c}, \delta p_{c}\right]$. Again the ratchet effect proves to be robust, as can be deduced from Fig. 7. The results of this figure are in agreement with the previous observation that the dependence of the ratchet current $\langle p\rangle$ on the cutoff value $p_{c}$ is weak (under the condition $p_{c} \gg k$ ).

\section{CONCLUSIONS}

Considering a realistic experimental scenario, we showed that a ratchet effect-induced by a combination of a twokick sequence as applied to an open system-is observable in an atom-optics kicked rotor experiment. We also checked the robustness of the ratcheted atomic evolution under reasonable noise conditions.

An interesting perspective would be to study the ratchet dynamics in a kicked Bose-Einstein condensate. Strong kicks may, however, lead to thermal excitations out of equilibrium and destroy the condensate, rendering the description by the usually applied Gross-Pitaevskii equation meaningless [22]. We have verified that the ratchet evolution is preserved in the presence of typical experimental nonlinearities. However, a full treatment of a strongly kicked Bose-Einstein condensate remains a challenge for future work.

\section{ACKNOWLEDGMENTS}

We gratefully acknowledge support by the MIUR COFIN2004 and 2005, the EU Specific Targeted Research Project OLAQUI, and the Alexander von Humboldt Foundation (Feodor-Lynen Program). G.G.C. gratefully acknowledges support by Conicet (Argentina).
[1] G. Casati, B. Chirikov, J. Ford, and F. Izrailev, in Stochastic Behavior in Classical and Quantum Hamiltonian Systems, edited by G. Casati and J. Ford (Springer, Berlin, 1979), p. 334.

[2] S. Fishman, in Quantum Chaos, School "E. Fermi" CXIX, edited by G. Casati, I. Guarneri, and U. Smilansky (IOS, Amsterdam, 1993).

[3] F. L. Moore, J. C. Robinson, C. F. Bharucha, B. Sundaram, and M. G. Raizen, Phys. Rev. Lett. 75, 4598 (1995); H. Ammann, R. Gray, I. Shvarchuck, and N. Christensen, ibid. 80, 4111 (1998); J. Ringot, P. Szriftgiser, J. C. Garreau, and D. Delande, ibid. 85, 2741 (2000); M. B. d'Arcy, R. M. Godun, G. S. Summy, I. Guarneri, S. Wimberger, S. Fishman, and A. Buchleitner, Phys. Rev. E 69, 027201 (2004); S. A. Wayper, W. Simpson, and M. D. Hoogerland, quant-ph/0602081 (unpublished); J. F. Kanem, S. Maneshi, M. Partlow, M. Spanner, and S. M. Steinberg, quant-ph/0604110 (unpublished).

[4] C. F. Bharucha, J. C. Robinson, F. L. Moore, B. Sundaram, Q. Niu, and M. G. Raizen, Phys. Rev. E 60, 3881 (1999); M. Sadgrove, S. Wimberger, S. Parkins, and R. Leonhardt, Phys. Rev. Lett. 94, 174103 (2005).

[5] G. G. Carlo, G. Benenti, G. Casati, and D. L. Shepelyansky, Phys. Rev. Lett. 94, 164101 (2005).

[6] T. S. Monteiro, P. A. Dando, N. A. C. Hutchings, and M. R. Isherwood, Phys. Rev. Lett. 89, 194102 (2002); T. Jonckheere, M. R. Isherwood, and T. S. Monteiro, ibid. 91, 253003 (2003);
E. Lundh and M. Wallin, ibid. 94, 110603 (2005).

[7] G. J. Duffy, S. Parkins, T. Muller, M. Sadgrove, R. Leonhardt, and A. C. Wilson, Phys. Rev. E 70, 056206 (2004).

[8] G. J. Duffy, A. S. Mellish, K. J. Challis, and A. C. Wilson, Phys. Rev. A 70, 041602(R) (2004).

[9] S. Wimberger, R. Mannella, O. Morsch, and E. Arimondo, Phys. Rev. Lett. 94, 130404 (2005).

[10] C. Ryu, M. F. Andersen, A. Vaziri, M. B. d'Arcy, J. M. Grossman, K. Helmerson, and W. D. Phillips, Phys. Rev. Lett. 96, 160403 (2006).

[11] R. Graham, M. Schlautmann, and P. Zoller, Phys. Rev. A 45, R19 (1992).

[12] P. H. Jones, M. M. Stocklin, G. Hur, and T. S. Monteiro, Phys. Rev. Lett. 93, 223002 (2004); G. Hur, C. E. Creffield, P. H. Jones, and T. S. Monteiro, Phys. Rev. A 72, 013403 (2005); H. Lignier, J. Chabé, D. Delande, J. C. Garreau, and P. Szriftgiser, Phys. Rev. Lett. 95, 234101 (2005).

[13] W. Ketterle, D. S. Durfee, and D. M. Stamper-Kurn, in BoseEinstein Condensation in Atomic Gases, Proceedings of the International School of Physics "Enrico Fermi," Course CXL edited by M. Inguscio, S. Stringari, and C. E. Wieman (IOS Press, Amsterdam, 1999), pp. 67-176.

[14] M. Kasevich, D. S. Weiss, E. Riis, K. Moler, S. Kasapi, and S. Chu, Phys. Rev. Lett. 66, 2297 (1991).

[15] S. Stock, Z. Hadzibabic, B. Battelier, M. Cheneau, and J. Dali- 
bard, Phys. Rev. Lett. 95, 190403 (2005), and references therein.

[16] W. H. Oskay, D. A. Steck, and M. G. Raizen, Chaos, Chaos, Solitons Fractals 16, 409 (2003).

[17] S. Wimberger and M. Sadgrove, J. Phys. A 38, 10549 (2005).

[18] A. Browaeys, H. Häffner, C. McKenzie, S. L. Rolston, K. Helmerson, and W. D. Phillips, Phys. Rev. A 72, 053605 (2005).

[19] S. Wimberger, I. Guarneri, and S. Fishman, Nonlinearity 16,
1381 (2003).

[20] T. Dittrich and R. Graham, Ann. Phys. (N.Y.) 200, 363 (1990).

[21] S. Flach, O. Yevtushenko, and Y. Zolotaryuk, Phys. Rev. Lett. 84, 2358 (2000); O. Yevtushenko, S. Flach, and K. Richter, Phys. Rev. E 61, 7215 (2000).

[22] S. A. Gardiner, D. Jaksch, R. Dum, J. I. Cirac, and P. Zoller, Phys. Rev. A 62, 023612 (2000); C. Zhang, J. Liu, M. G. Raizen, and Q. Niu, Phys. Rev. Lett. 92, 054101 (2004). 